\title{
A MID-INFRARED STUDY OF THE CLASS 0 CLUSTER IN LDN 1448
}

\author{
JoAnn C. O'Linger ${ }^{1}$ \\ Spitzer Science Center, California Institute of Technology, MS 314-6, \\ Pasadena, CA 91125; joanno@ipac.caltech.edu \\ David M. Cole ${ }^{1}$ and Michael E. Ressler \\ Jet Propulsion Laboratory, 4800 Oak Grove Drive, MS 306-388, Pasadena, CA 91109; \\ david.m.cole@jpl.nasa.gov, michael.e.ressler@jpl.nasa.gov \\ AND \\ Grace Wolf-Chase ${ }^{2}$ \\ Department of Astronomy and Astrophysics, University of Chicago, 5640 South Ellis Avenue, \\ Chicago, IL 60637; grace@horta.uchicago.edu \\ Received 2002 April 11; accepted 2005 December 16
}

\begin{abstract}
We present ground-based mid-infrared observations of Class 0 protostars in LDN 1448. Of the five known protostars in this cloud, we detected two, L1448N:A and L1448C, at 12.5, 17.9, 20.8, and 24.5 $\mu$ m, and a third, L1448 IRS 2, at $24.5 \mu \mathrm{m}$. We present high-resolution images of the detected sources and photometry or upper limits for all five Class 0 sources in this cloud. With these data we are able to augment existing spectral energy distributions for all five objects and place them on an evolutionary status diagram.
\end{abstract}

Key words: infrared: stars — ISM: individual (LDN 1448) — methods: data analysis (mid-infrared imaging) — stars: formation

\section{INTRODUCTION}

The majority of young stellar objects (YSOs) are classified using a scheme based on characteristics of their spectral energy distributions (SEDs) introduced by Lada \& Wilking (1984; see also Lada 1987, 1991). They proposed three classes: I, II, and III, distinguished from one another by their near- to mid-infrared spectral indices, which were presumed to be indicators of the evolutionary status of the object in question. Class I sources were initially thought to be representative of the earliest stage of premain-sequence evolution, i.e., protostars.

The spectral index, $\alpha$, is defined as

$$
\alpha=-\frac{d \log \nu F_{\nu}}{d \log \nu} ;
$$

for purposes of YSO classification, the region of the SED usually used for this calculation is from 2.2 to $10 \mu \mathrm{m}$. The spectral index is the negative of the slope of the SED when graphed in the frequency domain (Lada 1991).

The most current version of this slope classification scheme is presented in Greene et al. (1994). For a Class I source, the SED tends to be broader than a single-temperature blackbody and $\alpha>0.3$ due to the fact that the peak of the SED of such a source is shifted toward the far-infrared because of the very low effective temperature of the YSO. These sources are usually deeply embedded in their nascent dust envelopes. For Class II and Class III YSOs, on the other hand, $-0.3>\alpha \geq-1.6$ and $\alpha<-1.6$, respectively. The SED of a Class II YSO will be much broader than that of a Class III YSO due to the presence of circumstellar

\footnotetext{
${ }^{1}$ Visiting Astronomer at the Infrared Telescope Facility, which is operated by the University of Hawaii under contract from the National Aeronautics and Space Administration.

${ }^{2}$ Joint appointment at Adler Planetarium and Astronomy Museum, 1300 South Lake Shore Drive, Chicago, IL 60605.
}

dust with a fairly wide temperature distribution. Greene et al. (1994) identify a fourth class of "flat spectrum" sources, for which $0.3>\alpha \geq-0.3$, that have near-infrared spectra that are strongly veiled by continuum emission from hot circumstellar dust.

Observations of candidate protostars, in particular VLA 1623 in $\rho$ Oph A, an extremely cold $\left(T_{\text {eff }} \leq 20 \mathrm{~K}\right)$ object that does not appear at IRAS wavelengths, led to the introduction of a new class of YSOs by André et al. (1993). They contended that Class I sources could not be the youngest examples of pre-mainsequence objects (i.e., not true protostars) and promptly created a new category for such objects, designated Class 0 . The search for these protostars has gained impetus in the last several years due to the development of highly sensitive submillimeter continuum detectors.

Class 0 protostars are the rarest of YSOs. Pre-main-sequence stars typically spend $\ll 10^{5} \mathrm{yr}$ in this phase, on their way toward the onset of hydrogen fusion and stellar birth. The current set of defining characteristics of a Class 0 protostar are as follows (André et al. 2000):

1. Detection of a radio continuum source, presence of a molecular outflow, or some other evidence of a central protostellar source.

2. Centrally peaked but extended submillimeter continuum emission indicating an envelope as opposed to a mere disk.

3. A ratio of $L_{\text {submm }} / L_{\text {bol }}>0.5 \%$ (where $L_{\text {submm }}$ is the luminosity radiated by the object at wavelengths longward of $350 \mu \mathrm{m}$ and $L_{\text {bol }}$ is the total luminosity of the object).

This last criterion indicates a circumstellar mass greater than the mass of the central protostellar core, as discussed in André et al. (1993). There is no mention of the spectral index between 2 and $10 \mu \mathrm{m}$, since these sources have generally not been detected shortward of $10 \mu \mathrm{m}$ using ground-based instruments. This will no doubt change as detector technology evolves. 


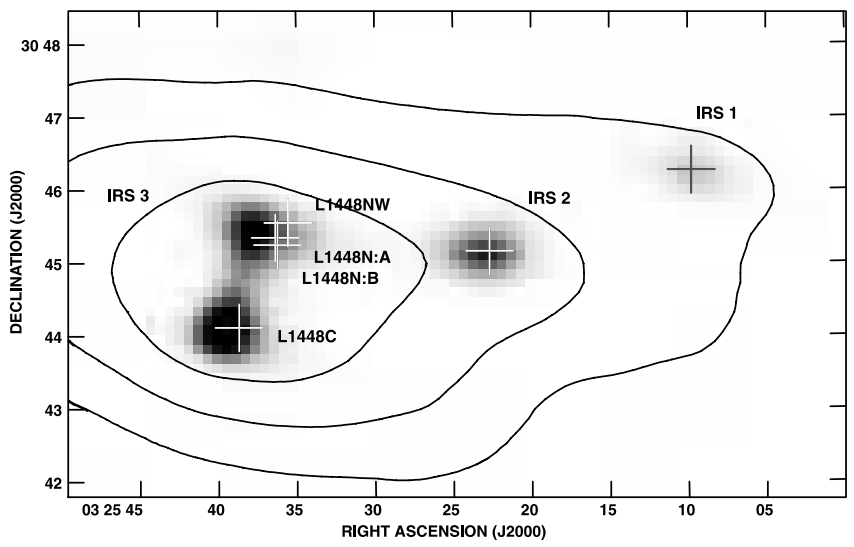

FIG. 1.-IRAS image showing the three Point Source Catalog objects in L1448, also known as L1448 IRS 1, IRS 2, and IRS 3. Contours represent the unenhanced co-added IRAS data, overlaid on the gray-scale plot of the corresponding HIRES data. Crosses indicate the known positions of YSOs from high-resolution observations at other wavelengths. The crosses are labeled with the most commonly used nomenclature for these sources found in the literature.

Although reports of newly discovered Class 0 protostars have increased dramatically in the last few years, confirmed sources in this category still number well under a hundred (André et al. 2000; Froebrich 2005). The SED of a Class 0 object, with a typical effective temperature of $\leq 40 \mathrm{~K}$, peaks at around $100 \mu \mathrm{m}$; until fairly recently, essentially the only tool with which one may study the Wien side of the SEDs of protostars has been the IRAS database.

The majority of known Class 0 sources were detected by IRAS at $25 \mu \mathrm{m}$, if not at $12 \mu \mathrm{m}$; however, due to the nature of the IRAS data, the effective beam size at mid-infrared wavelengths is never less than $\sim 30^{\prime \prime}-40^{\prime \prime}$, even when using the high-resolution data-processing program YORIC (Aumann et al. 1990; Hurt \& Barsony 1996; Barsony et al. 1998; O’Linger et al. 1999). Figure 1 provides an example of co-added $60 \mu \mathrm{m}$ IRAS data before (contours) and after (gray scale) HIRES processing via YORIC. The poor spatial resolution of the IRAS data makes associating point sources in the catalog with data from other wavelengths difficult and, in cases of close binary or multiple systems, does not allow the contributions of the individual components to the total flux to be distinguished. HIRES point-source modeling, which makes use of a little-known feature of the YORIC program, has been used successfully to estimate individual IRAS fluxes from sources as close as $20^{\prime \prime}$ apart, but this still does not compare with the subarcsecond resolution obtainable with millimeter-wave interferometry (Barsony et al. 1998). Consequently, the mid-infrared SEDs of Class 0 sources have tended to be poorly constrained. Data from the latest NASA Great Observatory, the Spitzer Space Telescope, are already contributing significantly to our understanding of the mid-IR characteristics of YSOs, but Spitzer lacks the resolution to augment the information on the small-scale structure of their circumstellar environments, which may be obtained from submillimeter- and millimeter-wavelength observations (Chandler \& Richer 2000; Looney et al. 2000).

Some of the most important questions in star formation studies today center around the multiplicity of these young systems and how the binary properties evolve with time (Looney et al. 2000; Reipurth 2000). The majority of nearby young T Tauri stars are known to be binary systems, yet only a very few Class 0 protobinary systems have been resolved. It has been postulated that the giant Herbig-Haro flows driven by many YSOs may be initiated by the dynamical decay of unstable multiple systems
(Reipurth 2000). Understanding star formation in the context of multiple systems has important implications for understanding the initial mass function and its physical meaning.

In order to address the issue of multiplicity and obtain details about the immediate circumstellar environments of Class 0 sources, it is necessary to study these objects at as many wavelengths as possible with high angular resolution.

A particularly rich cluster of Class 0 sources is located in the compact globule L1448, a molecular cloud that is part of the Perseus molecular complex. L1448 lies at a distance of $\sim 300 \mathrm{pc}$; its mass, estimated from ammonia data, is $\sim 50 M_{\odot}$ (Bachiller \& Cernicharo 1986a). Three embedded infrared sources were detected by IRAS in this cloud; since then, it has been surveyed at several wavelengths and mapped in a number of molecular lines (Bachiller \& Cernicharo 1986b; Anglada et al. 1989; Bally et al. 1997; Eislöffel 2000; Wolf-Chase et al. 2000). Listed from west to east, the IRAS Point Source Catalog designations for the three sources are IRAS $03220+3035$, IRAS $03222+3034$, and IRAS $03225+3034$. In the literature, these objects are more commonly known as L1448 IRS 1, L1448 IRS 2, and L1448 IRS 3, respectively (see Fig. 1).

Only the westernmost of these objects (IRS 1) has been detected at near-infrared and shorter wavelengths (Cohen \& Kuhi 1979; Neckel \& Staude 1984); this source has been identified as a Class I YSO and hence was not included in our survey (Eislöffel 2000; M. Barsony 2000, private communication). The other two IRAS point sources have been found to contain multiple Class 0 objects. IRS 2 was recently reported by Wolf-Chase et al. (2000) to be a candidate protobinary system based on the two distinct molecular outflows that emerge from that position, although the binary components were unresolved at that time. Subsequently, Volgenau et al. (2002) announced that both components of the IRS 2 binary had in fact been detected at BIMA. IRS 3 consists of L1448N:A, B (a 7" protobinary), L1448NW, and L1448C (note that L1448C is frequently referred to as L1448-mm), all of which drive molecular outflows as well (Terebey \& Padgett 1997; Barsony et al. 1998; Eislöffel 2000; Wolf-Chase et al. 2000). The locations of all of these sources, from previously published high-resolution submillimeter- or millimeter-wave observations, are indicated by crosses in Figure 1 (O'Linger et al. 1999; Looney et al. 2000; André et al. 2000, and references therein).

We observed all of the known L1448 Class 0 protostars in the mid-infrared and present images of the detected sources plus multiwavelength photometry and/or upper limits for all five Class 0 sources.

\section{OBSERVATIONS AND DATA REDUCTION}

Low-mass Class 0 objects are generally not visible at wavelengths shorter than $10 \mu \mathrm{m}$ using current (ground-based) technology (André et al. 1993; Barsony 1994). The shortest wavelengths at which these sources are generally thought to be easily detectable are in the $\lambda \sim 20 \mu \mathrm{m}$ atmospheric window. MIRLIN, the Jet Propulsion Laboratory's $128 \times 128$ pixel Si:As mid-infrared camera (Ressler et al. 1994), provides access to this wavelength regime.

The observations reported here were carried out using MIRLIN at NASA's $3 \mathrm{~m}$ Infrared Telescope Facility (IRTF) on the nights of 2000 February 18-19 UT and 2000 October 5 UT. For these observations the measured pixel scale was 0.482 , yielding a field of view just over $1^{\prime}$ on a side. To remove the thermal background signal from the sky and the telescope, the secondary mirror was chopped $28^{\prime \prime}$ southeast-northwest at about a $5 \mathrm{~Hz}$ rate and the telescope nodded the same distance and direction every few hundred chop cycles. The L1448C source and the L1448N/NW 
system were both observed at $12.5,17.9,20.8$, and $24.5 \mu \mathrm{m}$, while L1448 IRS 2 was observed using only the latter two filters. The sources $\alpha$ Aur, $\alpha$ Boo, and $\alpha$ CMi were observed throughout each night in February in all four passbands, both to serve as photometric calibrators and to track the weather conditions and air-mass dependence. In October, $\alpha$ Lyr was used for the same purposes. The weather was excellent in February but more variable in October.

The data were reduced using our in-house IDL routine MAC (match and combine), which performs the background subtraction, identification, and shifting of the source peaks and the coaddition of the registered data. Circular aperture photometry was then performed on both the standards and the L1448 sources using apertures of radius 5 pixels (2".4).

\section{RESULTS}

Our observations of L1448 IRS 3 are presented in Figures 2 and 3; each of the four panels is labeled with the central wavelength of the filter used and has an image of the point-spread function (PSF) at the appropriate wavelength in the lower left corner. Figure 2 shows our observations of L1448C, which at these resolutions $\left(00^{\prime \prime} 90,11^{\prime \prime} 29,1{ }^{\prime \prime} 50\right.$, and 1 .".77 at 12.5, 17.9, 20.8, and $24.5 \mu \mathrm{m}$, respectively) is indistinguishable from an isolated point source. Figure 3 shows our images of the rest of the sources in IRS 3, all of which lie within a single MIRLIN field of view: the $7^{\prime \prime}$ binary, L1448N:A, B, and a third source $\sim 20^{\prime \prime}$ away, L1448NW (Terebey \& Padgett 1997; Barsony et al. 1998; WolfChase et al. 2000; Eislöffel 2000; Looney et al. 2000). Although there appears to be a hint of extended emission around L1448N:A in the 20.8 and $24.5 \mu \mathrm{m}$ images, the extension is northwest/ southeast, in good alignment with the direction of the chop, and therefore is most likely an artifact of the chop pattern.

In 2000 February we detected a single source in the L1448N/ NW field at the location of L1448N:A; this was initially somewhat surprising, since N:B is actually quite a bit brighter than $\mathrm{N}: \mathrm{A}$ at millimeter wavelengths. We therefore made additional observations of this field in 2000 October. Using the nearby bright star BS 999 as a position reference, we find that the single

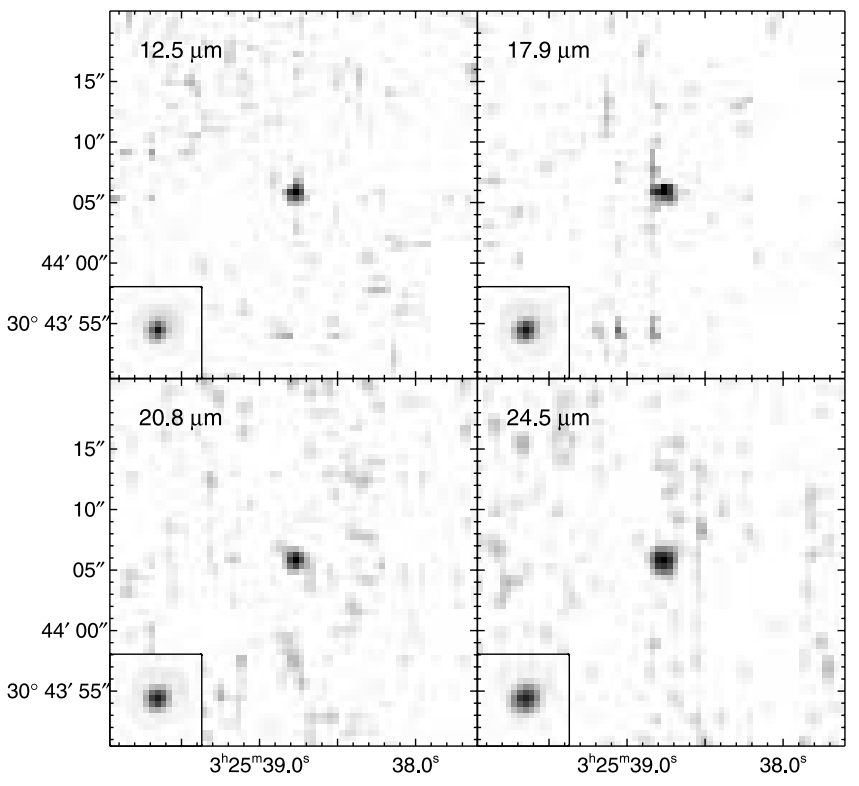

FIG. 2.-MIRLIN images of L1448C. Each panel is labeled with the corresponding central wavelength of the filter used. The source is clearly detected and indistinguishable from a point source at all wavelengths. The image field of view is $30 . " 8 \times 30 "$ " 8 .

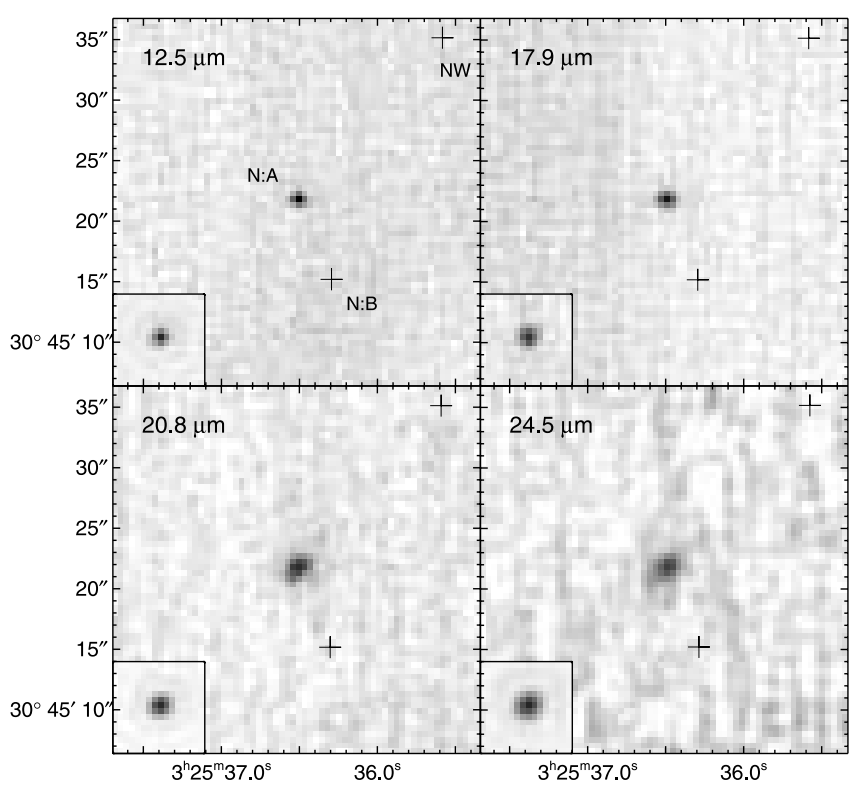

FIG. 3.-MIRLIN images of L1448N. Each panel is labeled with the corresponding central wavelength of the filter used. The detected source is L1448N:A; crosses indicate the positions of other YSOs (L1448N:B, L1448NW) in the field that were not detected. Faint elongations seen in these images are almost certainly artifacts of the chop pattern. The image field of view is $30.8 \times 30^{\prime \prime} 8$.

source is consistent only with L1448N:A; for it to be N:B or NW would require telescope positioning errors of 7".3 and 17".5 (15 and 37 pixels), respectively. In Figure 3, crosses show the positions at which N:B and NW should appear. These results agree with those presented by Ciardi et al. (2003) for the L1448N:A, B system.

Figure 4 shows the MIRLIN image of L1448 IRS 2 at $24.5 \mu \mathrm{m}$, along with the PSF at that wavelength. IRS 2 was observed at 20.8 and $24.5 \mu \mathrm{m}$ but detected (at $4 \sigma$ ) only at the longer wavelength.

Table 1 provides the coordinates of each source and presents the MIRLIN photometry and upper limits. Coordinates for the

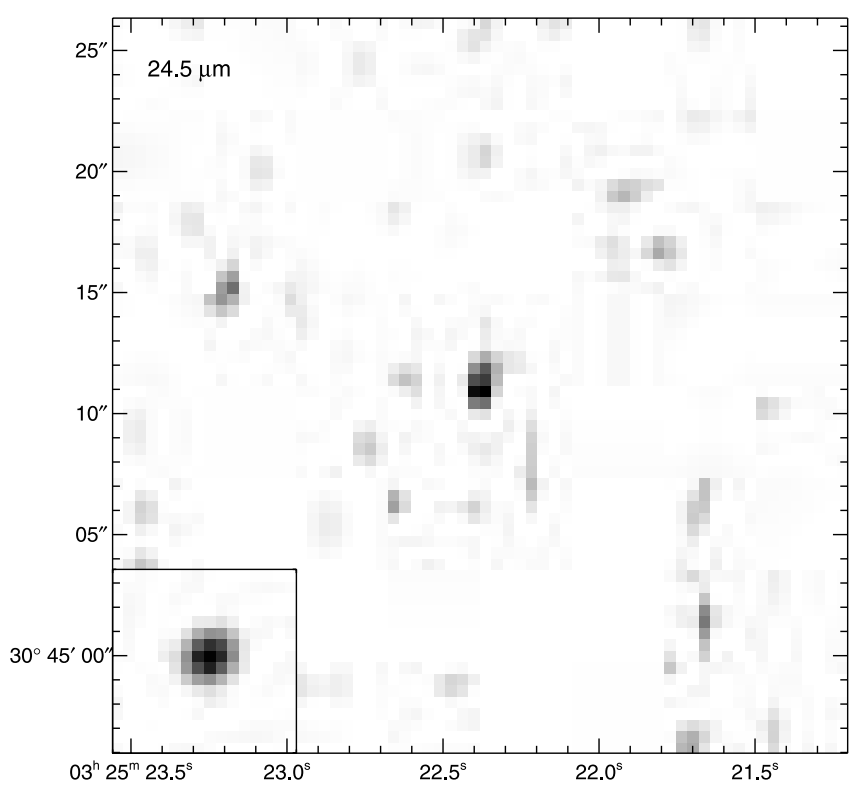

FIG. 4.-MIRLIN image of L1448 IRS 2 at $24.5 \mu \mathrm{m}$. The source was marginally detected at this wavelength and undetected at $20.8 \mu \mathrm{m}$. We did not attempt to observe it in any other filters. The image field of view is 30 " $8 \times 30$ " 8 . 
TABLE 1

Source List AND MIRLIN Photometry

\begin{tabular}{|c|c|c|c|c|c|c|}
\hline Source & R.A. & Decl. & $\begin{array}{c}F_{12.5} \\
(\mathrm{Jy})\end{array}$ & $\begin{array}{c}F_{17.9} \\
(\mathrm{Jy})\end{array}$ & $\begin{array}{c}F_{20.8} \\
(\mathrm{Jy})\end{array}$ & $\begin{array}{c}F_{24.5} \\
(\mathrm{Jy})\end{array}$ \\
\hline L1448 IRS $2 \ldots \ldots \ldots \ldots$ & 032522.4 & +304512 & $\ldots$ & $\ldots$ & $<0.46$ & $0.64 \pm 0.23$ \\
\hline 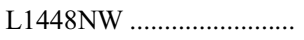 & 032535.653 & +304534.20 & $<0.15$ & $<0.30$ & $<0.27$ & $<1.85$ \\
\hline 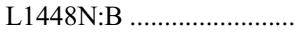 & 032536.339 & +304514.94 & $<0.15$ & $<0.30$ & $<0.27$ & $<1.85$ \\
\hline L1448N:A .......................... & 032536.532 & +304521.35 & $0.43 \pm 0.10$ & $2.62 \pm 1.54$ & $2.80 \pm 0.65$ & $5.44 \pm 1.80$ \\
\hline L1448C ….............................. & 032538.8 & +304405.0 & $0.29 \pm 0.04$ & $0.73 \pm 0.14$ & $0.55 \pm 0.17$ & $2.37 \pm 1.52$ \\
\hline
\end{tabular}

Notes.- Units of right ascension are hours, minutes, and seconds, and units of declination are degrees, arcminutes, and arcseconds. All upper limits are $3 \sigma$.

Class 0 sources L1448N:A, B and NW were obtained from Looney et al. (2000); the positions of L1448C and L1448 IRS 2 were taken from André et al. (2000). Errors in the photometry are dominated by the calibration uncertainties; even the most marginal detection, IRS 2, was $4 \sigma$. For L1448N:A and L1448C, signal-to-noise ratios varied between 6 and 23 .

Figures 5-8 present SED plots for the three detected objects (L1448C, L1448N:A, and L1448 IRS 2), as well as for the undetected sources L1448N:B and L1448NW, constrained in the mid-infrared by the MIRLIN upper limits (arrows). L1448NW, which is shown in Figure 7 for completeness, was not detected, and we were unable to improve on previous upper limits in the mid-infrared that were derived from HIRES point-source modeling (Barsony et al. 1998). Previously reported data from the literature are plotted in combination with our MIRLIN fluxes. The SEDs are shown with either single- or dual-graybody fits to the data. Those SED figures with dual-graybody fits (solid lines) also display single-temperature graybodies (dashed lines), each graybody being a modified blackbody of the form

$$
S_{\nu}=B_{\nu}\left(T_{d}\right)\left(1-e^{-\tau_{\nu}}\right) d \Omega,
$$

where $B_{\nu}\left(T_{d}\right)$ is the Planck function at frequency $\nu$ and dust temperature $T_{d}, \tau_{\nu}$ is the dust optical depth, and $d \Omega$ is the solid

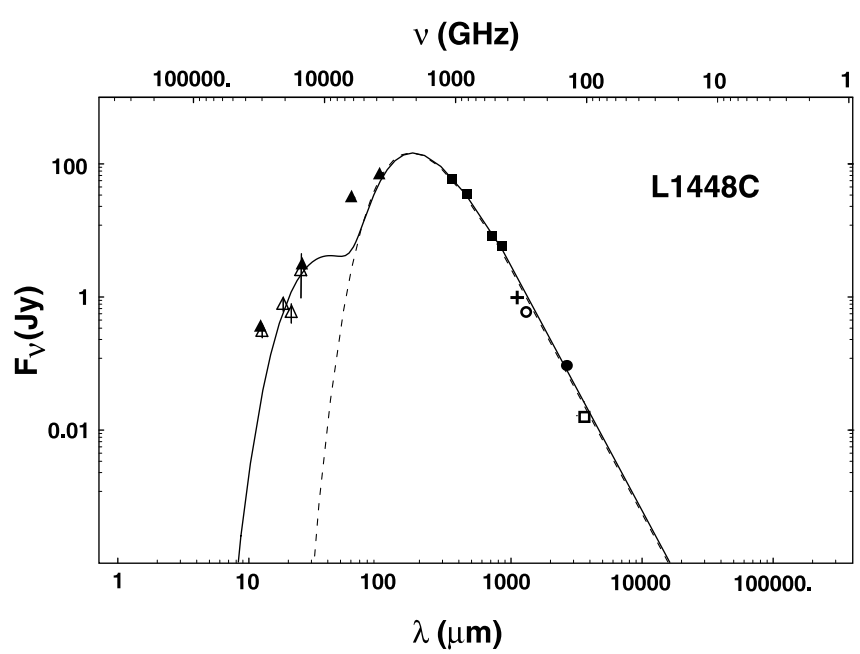

Fig. 5.-SED of L1448C. The flux data for L1448C are presented, along with a single-temperature graybody fit (dashed line) and a dual-graybody fit (solid line). Some of the parameters of the dual-graybody fit are presented in Table 2. The filled triangles represent IRAS photometry from HIRES point-source modeling (Barsony et al. 1998); the open triangles with error bars are from MIRLIN observations (this work). Filled squares represent James Clerk Maxwell Telescope (JCMT) data from Chandler \& Richer (2000), the plus sign represents an IRAM flux from Barsony et al. (1998), and the open circle is from Bachiller et al. (1991). The filled circle indicates flux data from Bachiller et al. (1995), while the open square is from Guilloteau et al. (1992). angle subtended by the source. The sole exception to this convention is Figure 6, which presents fits for the data from each component of the $\mathrm{L} 1448 \mathrm{~N}$ protobinary using a dashed line for $\mathrm{N}: \mathrm{A}$ and a dotted line for $\mathrm{N}: \mathrm{B}$, while the solid line represents the sum of those two individual SED fits; note that this particular pair of fits to the data is not the only possible solution, and this fact is discussed at length in $\S 4.1 .2$.

The various dual-graybody model fits to the data, shown by the solid lines in Figures 5-8, were produced by simply summing together two graybody curves, each with a different effective dust temperature. It is clearly not possible to fit all of the data points for any of the sources that were detected by MIRLIN in the mid-infrared with a single-temperature graybody. The dualgraybody curves yield more accurate calculations of the bolometric luminosities. The model fits to the flux data for each source and any necessary assumptions are discussed in detail in $\S 4$.

Table 2 lists each source, the number of graybodies $\left(N_{\mathrm{gb}}\right)$ used to fit the flux data, the associated dust temperatures $\left(T_{d}\right)$ for each

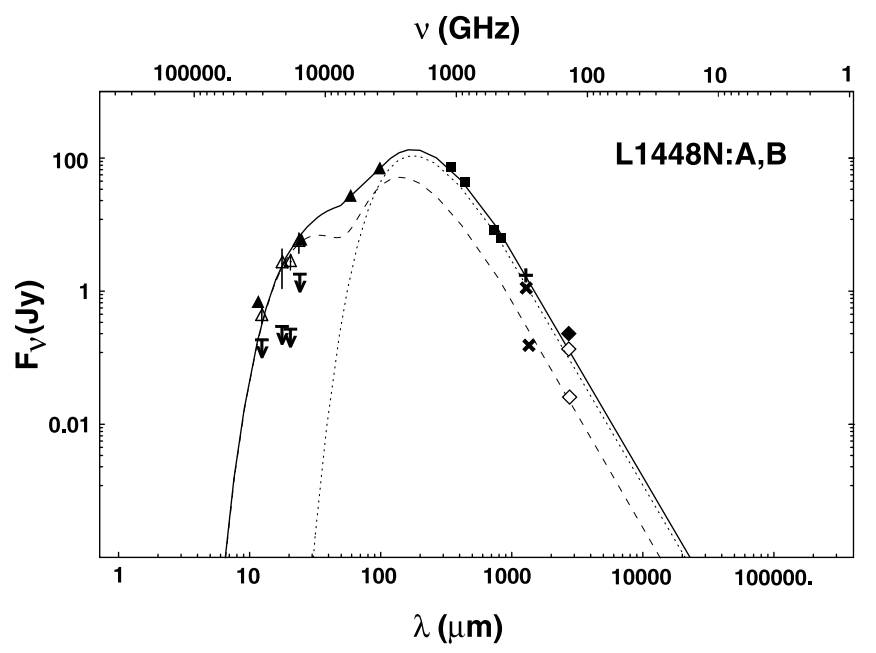

FIG. 6.-SEDs of the L1448N protobinary components. The individual flux data for the two protobinary components, $\mathrm{L} 1448 \mathrm{~N}$ :A and N:B, are presented in conjunction with the literature flux values for the total emission from the L1448N binary at various wavelengths. The fits to the individual fluxes shown for each source (dashed and dotted lines, fits for $\mathrm{N}: \mathrm{A}$ and $\mathrm{N}: \mathrm{B}$, respectively) were generated assuming that each component emits approximately the same flux at $100 \mu \mathrm{m}$ (i.e., case 3 ; see $\S 4.1 .2$ for a discussion). Some of the parameters of the single- and dual-graybody fits are presented in Table 2. The solid line represents the sum of the fits to the individual flux data points (dashed and dotted lines). The filled triangles represent IRAS photometry from HIRES pointsource modeling (Barsony et al. 1998); the open triangles with error bars and the upper limits are from MIRLIN observations (this work). Squares indicate JCMT data from Chandler \& Richer (2000), and the plus sign represents IRAM data from Barsony et al. (1998). The crosses indicate fluxes from Terebey \& Padgett (1997). The open diamonds are from Looney et al. (2000), while the filled diamond is the sum of those fluxes. 


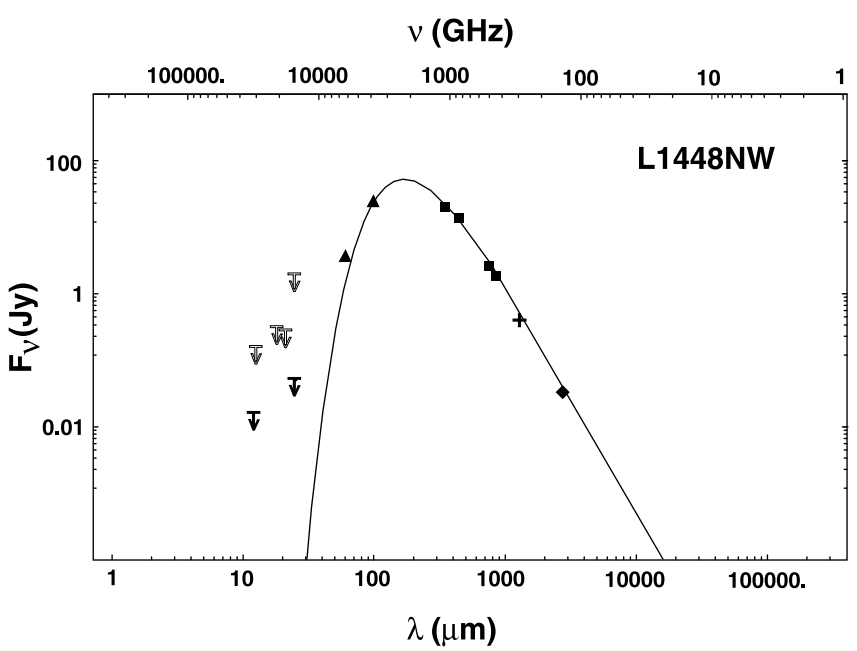

FIg. 7.-SED of L1448NW. The flux data for L1448NW are presented, along with a single-temperature graybody fit (solid line). Some of the parameters of the single-graybody fit are presented in Table 2. The filled upper limits and the triangles represent IRAS photometry from HIRES point-source modeling (Barsony et al. 1998); the open upper limits are from MIRLIN observations (this work). Squares indicate JCMT data from Chandler \& Richer (2000), the plus sign represents IRAM data from Barsony et al. (1998), and the diamond is from Looney et al. (2000).

graybody, and two physical parameters derived for these sources from the SED fitting: the bolometric luminosity $L_{\text {bol }}$ and the infall envelope mass $M_{\text {env }}$, estimated from (Hildebrand 1983)

$$
M_{\mathrm{env}}=\frac{S_{\lambda} d^{2}}{\kappa_{\lambda} B_{\lambda}\left(T_{d}\right)},
$$

evaluated at $\lambda=1.3 \mathrm{~mm}$.

Recall that dust emission is generally optically thin at millimeter wavelengths and is therefore a direct indicator of the total mass (gas + dust) contained in molecular cloud cores given appropriate assumptions about the dust-to-gas ratio. The appropriate value of $\kappa_{1.3 \mathrm{~mm}}$ to use depends on the status or "age" of the YSOs in the

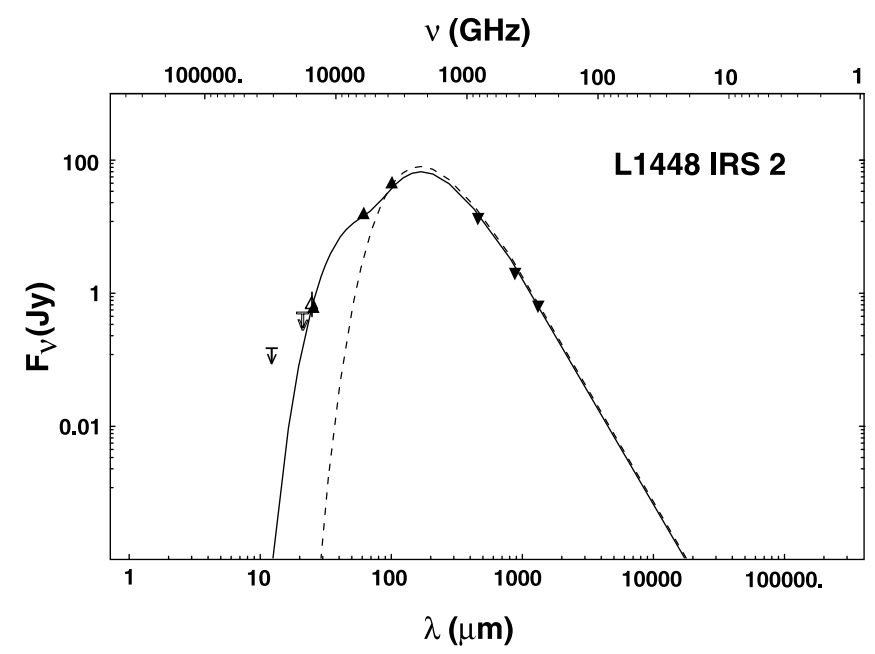

FIG. 8.-SED of L1448 IRS 2. The flux data for IRS 2 are presented, along with a dual-graybody fit (solid line) and a single-temperature graybody fit (dashed line). Some of the parameters of the dual-graybody fit are presented in Table 2. The filled upper limit and the filled upward triangles represent IRAS photometry from HIRES point-source modeling (O'Linger et al. 1999); the open triangle with the error bar and the open upper limit are MIRLIN data (this work). The filled downward triangles indicate photometry obtained with SCUBA at the JCMT and $1.3 \mathrm{~mm}$ continuum flux data from the $12 \mathrm{~m}$ millimeterwave dish at Kitt Peak (O’Linger et al. 1999).
TABLE 2

Derived Physical Parameters

\begin{tabular}{|c|c|c|c|c|c|}
\hline Source & $N_{\mathrm{gb}}$ & $\begin{array}{c}T_{d} \\
(\mathrm{~K})\end{array}$ & $\begin{array}{l}L_{\text {bol }} \\
\left(L_{\odot}\right)\end{array}$ & $\begin{array}{l}M_{\mathrm{env}} \\
\left(M_{\odot}\right)\end{array}$ & $\begin{array}{c}L_{\text {submm }} / L_{\text {bol }} \\
(\%)\end{array}$ \\
\hline L1448 IRS 2 . & 2 & 20,50 & 5.6 & 0.73 & 3.3 \\
\hline L1448NW & 1 & 20 & 3.5 & 0.68 & 5.0 \\
\hline $\mathrm{L} 1448 \mathrm{~N}: \mathrm{B}^{\mathrm{a}} \ldots$. & 1 & 17.5 & 5.5 & 1.75 & 7.3 \\
\hline 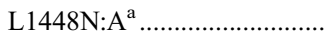 & 2 & 23,90 & 4.8 & 0.29 & 1.7 \\
\hline … & 2 & 19,85 & 10.0 & 1.10 & 3.7 \\
\hline
\end{tabular}

a Parameters for L1448N:A, B are derived by assuming approximately equal $100 \mu \mathrm{m}$ fluxes.

core in question due to the evolution of dust properties (André et al. 2000). We used $\kappa_{1.3 \mathrm{~mm}}=0.01$ based on literature values for similar regions (André et al. 1993; André \& Montmerle 1994).

In addition, the ratio of the submillimeter luminosity to the bolometric luminosity, $L_{\text {submm }} / L_{\text {bol }}$, where $L_{\text {submm }}$ is calculated using wavelengths longer than $350 \mu \mathrm{m}$, was determined for each source and included in Table 2 . This ratio can be used as a diagnostic tool to determine the evolutionary status of embedded YSOs because it is linked with the ratio of the circumstellar envelope mass to the mass of the central accreting core and thus decreases with time. A value of $L_{\text {submm }} / L_{\text {bol }}>0.5 \%$ indicates $M_{\text {env }} / M_{*}>1$, i.e., an envelope mass greater than the protostellar core mass, a distinguishing characteristic of Class 0 sources (see $\S 1$ ).

\section{DISCUSSION}

\subsection{Spectral Energy Distributions: Individual Sources}

\subsection{1. $L 1448 C$}

This Class 0 source, which drives a powerful, highly collimated outflow, is a favorite target of many observers (see Barsony et al. 1998 and references therein). When first discovered, it was believed to be one of the youngest Class 0 sources found, with a kinematic age (based on the outflow parameters) of $\sim 3500 \mathrm{yr}$ (Bachiller et al. 1990). This figure was revised upward to $\sim 32,000$ yr by Barsony et al. (1998), who found that the outflow was far more extensive than previously thought (see also WolfChase et al. 2000).

The SED for L1448C is presented in Figure 5, with a dualgraybody fit to the data shown by the solid line. The dashed line indicates a single-temperature graybody fit to the data.

\subsubsection{L1448N:A, $N: B$}

In order to analyze the SEDs for L1448N:A and N:B and use them to derive the parameters listed in Table 2, it is necessary to make certain assumptions regarding the individual fluxes of these two objects at far-infrared wavelengths. There are no high angular resolution data that can separate the $7^{\prime \prime}$ protobinary components at or near the peak of the dust emission $(\sim 100 \mu \mathrm{m})$, and this causes problems when attempting to fit modified blackbodies to the SEDs of these objects.

Due to the excellent correspondence between the 12 and $25 \mu \mathrm{m}$ IRAS HIRES point-source model fluxes from Barsony et al. (1998) for L1448N (which represent the sums of the fluxes for both components of the protobinary) and our MIRLIN fluxes (see Fig. 6), it seems clear that the mid-infrared fluxes are due chiefly to L1448N:A, with very little, if any, contribution from $\mathrm{N}: \mathrm{B}$ or NW. Again, these results agree with those presented in Ciardi et al. (2003). In the millimeter, the situation is quite different: N:B produces most of the flux seen at $2.7 \mathrm{~mm}$ (Terebey \& Padgett 1997; Looney et al. 2000). Using HIRES modeling, 
Barsony et al. (1998) showed that it is possible to separate the $I R A S$ fluxes produced by L1448NW from the total flux emitted by the L1448N triple system. The L $1448 \mathrm{~N}$ system produces a total of $89 \mathrm{Jy}$ at $100 \mu \mathrm{m}$, while the HIRES-modeled $100 \mu \mathrm{m}$ flux from NW is $\sim 23 \mathrm{Jy}$. Therefore, the maximum possible flux from the protobinary at $100 \mu \mathrm{m}$ must be on the order of 66 Jy. Since some of the emission detected by IRAS at that wavelength is presumably due to shock-heated dust caused by the impact of the L1448C outflow on the dense core containing the L1448N protobinary, the true $100 \mu \mathrm{m}$ flux from the binary is probably somewhat less than 66 Jy (Barsony et al. 1998; Curiel et al. 1999).

The flux ratio is approximately $5: 1$ in favor of $\mathrm{N}: \mathrm{B}$ in the millimeter regime (Looney et al. 2000); therefore, it seems reasonable to explore a range of possibilities for the $100 \mu \mathrm{m}$ fluxes. We have done so, in the following fashion: (1) Assume that the fluxes at $100 \mu \mathrm{m}$ have the same ratio as the $2.7 \mathrm{~mm}$ fluxes, so that N:A produces $11 \mathrm{Jy}$ and $\mathrm{N}: \mathrm{B}$ emits the remaining $55 \mathrm{Jy}$. (2) Assume the inverse scenario (i.e., N:B has a flux of $11 \mathrm{Jy}$, $\mathrm{N}$ :A produces the other $55 \mathrm{Jy}$ ). These two possibilities define the endpoints of the range that we consider in this paper. A third instructive case to consider is the intermediate scenario, which involves dividing the 66 Jy into equal portions; this assumes fluxes of $\sim 33 \mathrm{Jy}$ at $100 \mu \mathrm{m}$ from each of the protobinary components.

The assumptions inherent in this range of possibilities were used to generate a series of different SEDs for N:A and N:B; each of the SEDs for N:A were then fitted with dual-graybody models, while those for N:B were done with single-temperature graybodies. Sanity checks were performed for each case by using the various fits to calculate ranges of $L_{\text {bol }}$ for the two sources and thus to ascertain whether the sum of their luminosities approximates the total luminosity expected for the protobinary. The luminosity of the protobinary was derived by taking the total fluxes of the L1448N system reported in the literature and subtracting the flux data for L1448NW at all wavelengths, then plotting the resultant SED and fitting with the sum of two modified blackbodies; this yields a maximum expected $L_{\text {bol }}$ of $\sim 14.1 L_{\odot}$ for the protobinary system. The derivation of the L1448NW luminosity, using a single graybody fit to the data, is discussed in $\S 4.1 .3$.

From the analysis described above, we find that the plausible range of $L_{\text {bol }}$ for $\mathrm{L} 1448 \mathrm{~N}$ :A is $3.1-6.6 L_{\odot}$, and for $\mathrm{N}: \mathrm{B}$ it is $2.8-$ $7.4 L_{\odot}$. Since N:B dominates in the millimeter and N:A in the mid-infrared and the two objects have bolometric luminosities of the same order of magnitude, their SEDs must cross each other somewhere in the far-infrared, probably in the general vicinity of $100 \mu \mathrm{m}$. The scenario of approximately equal $100 \mu \mathrm{m}$ fluxes thus seems the most illustrative; it yields bolometric luminosities of $\sim 4.8 L_{\odot}$ for N:A and $5.5 L_{\odot}$ for $\mathrm{N}: \mathrm{B}$. The sum is indeed less than the maximum expected luminosity.

Figure 6 is a plot of the literature and MIRLIN flux data for each protobinary component along with a dual-graybody fit for L1448N:A (dashed line) and a single-temperature graybody fit for N:B (dotted line) using the "equal $100 \mu \mathrm{m}$ fluxes" scenario. The previously published data points and derived fluxes for the entire L1448N protobinary system are plotted, and the sum of the fits to the individual SEDs (i.e., dashed + dotted) is shown as a solid line. The values of the physical parameters derived from the assumption of approximately equal $100 \mu \mathrm{m}$ fluxes (case 3) are reported in Table 2 .

\subsection{3. $L 1448 N W$}

L1448NW was not detected by MIRLIN at any wavelength, as can be seen in Figure 3. This was the expected result due to the

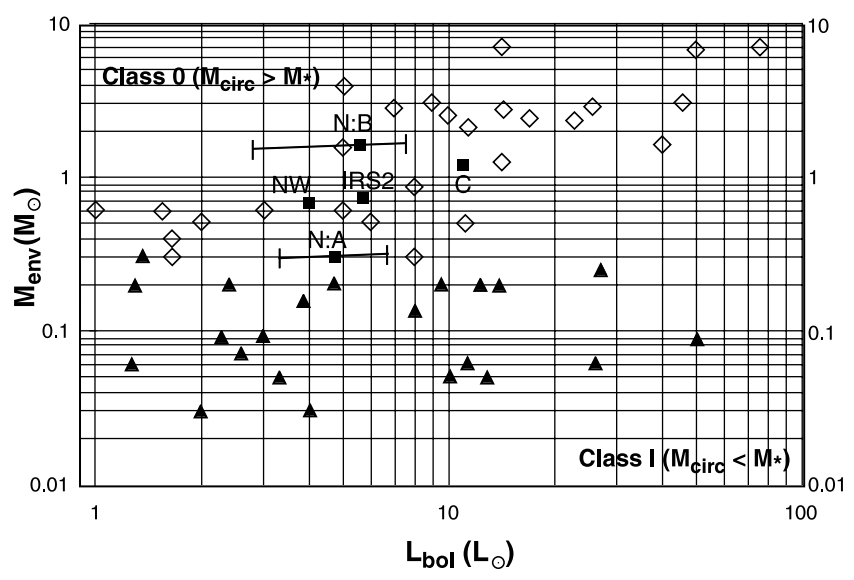

FIG. 9.-Evolutionary status diagram of the L1448 objects: $M_{\text {env }}$ vs. $L_{\text {bol }}$. We plot the positions of the L1448 protostars (squares) along with a number of other well-known Class 0 (diamonds) and Class I sources (triangles) from André et al. (2000) and Bontemps et al. (1996) in the circumstellar mass vs. bolometric luminosity diagram. Since bolometric luminosity is an indirect measure of central core mass, this is essentially a plot of envelope mass vs. central core mass. The "error bars" on L1448N:A and N:B represent the full range of likely values for each object (all three cases, with the filled square being case 3 ; see $\S 4.1 .2$ ). Generally, sources to the upper left of the diagram are in the Class 0 region, while sources to the lower right are Class I objects.

extensive HIRES point-source modeling work reported in Barsony et al. (1998), which established IRAS upper limits for this lowluminosity source of 0.015 and $0.05 \mathrm{Jy}$ at 12 and $25 \mu \mathrm{m}$, respectively. Our MIRLIN upper limits do not improve on these results (see Table 1).

We have incorporated the submillimeter- and millimeter-wave flux data from Chandler \& Richer (2000) and Looney et al. (2000) in the plot shown in Figure 7; these data are entirely consistent with the far-infrared IRAS fluxes derived from HIRES modeling (Barsony et al. 1998). The results from a single-temperature graybody fit to the data at wavelengths longer than $60 \mu \mathrm{m}$ are used to calculate the parameters reported in Table 2, which allow us to place L1448NW on the evolutionary diagram in Figure 9.

\subsection{4. $L 1448$ IRS 2}

L1448 IRS 2 was identified as a Class 0 source by O'Linger et al. (1999) and first proposed as a candidate protobinary in Wolf-Chase et al. (2000) based on CO observations that mapped two distinct molecular outflows driven by IRS 2. Volgenau et al. (2002) claimed detection of both binary components using BIMA, although the spatial separation was not reported. With the high spatial resolution of MIRLIN at the IRTF $\left(<2^{\prime \prime}\right.$ at all wavelengths), we had hoped to be able to detect and resolve both components of the system, but only a single source was found, at the $4 \sigma$ level in the $24.5 \mu \mathrm{m}$ filter (see Fig. 4). This suggests either that the binary components are too close together for MIRLIN to resolve (see the first paragraph of $\S 3$ for MIRLIN resolutions) or that the fluxes from one of the components are below the detection thresholds at all MIRLIN wave bands.

Figure 8 presents the SED for IRS 2, with a dual-graybody fit to the data shown by the solid line. The dashed line indicates a single-temperature graybody fit to the data.

\subsection{Evolutionary Status of L1448 Sources}

$$
\text { 4.2.1. } M_{\mathrm{env}} \text { versus } L_{\mathrm{bol}}
$$

Figure 9 is a plot of $M_{\text {env }}$ versus $L_{\text {bol }}$ for all of the sources in this paper (squares), along with all other Class 0 (diamonds) and 
Class I YSOs (triangles) with $L_{\text {bol }} 1.0$ from Table 1 in Bontemps et al. (1996) plotted for comparison. This plot can be used as a diagnostic tool for determining the relative ages of embedded sources (André \& Montmerle 1994; Saraceno et al. 1996). The diagram shows infall envelope mass (along the vertical axis) versus bolometric luminosity (horizontal axis). The value of $L_{\mathrm{bol}}$ is directly related to the mass of the central protostellar core at very early stages of evolution, assuming all luminosity is generated by gravitational infall, according to

$$
L_{\mathrm{bol}}=\frac{G \dot{M} M_{*}}{R_{*}},
$$

where a protostellar radius of $\sim 3 R_{\odot}$ is assumed (Stahler et al. 1980b).

Our results depicted in Figure 9 for L1448C and L1448NW are consistent (within errors) with those presented in Barsony et al. (1998) for those two sources. The new results in the diagram presented here are that (1) L1448 IRS 2 is plotted on the diagram to allow comparison with the other sources from this cloud, and (2) we have plotted the L1448N protobinary components separately on this graph, using the assumptions discussed in $\S$ 4.1.2.

All of the target objects discussed in this paper lie within the Class 0 region of the evolutionary diagram in Figure 9, although L1448N:A appears to be close to the transition zone between Class 0 and Class I, indicating N:A may be the most evolved source in this cloud after the Class I source L1448 IRS 1. By the same criteria, L1448N:B is very likely the youngest source that we attempted to image in this cloud. These classifications are reflected in the values of the parameter $L_{\text {submm }} / L_{\text {bol }}$, listed in Table 2 , which range from $1.7 \%$ to $7.3 \%$ : the value of $1.7 \%$ corresponds to $\mathrm{L} 1448 \mathrm{~N}: \mathrm{A}$ and $7.3 \%$ to $\mathrm{N}: \mathrm{B}$ (for the case 3 scenario of equal $100 \mu$ m fluxes).

We note that recently Ciardi et al. (2003) have argued that L1448N:A is probably a Class I object. However, our smallest value of $L_{\text {submm }} / L_{\text {bol }}$ for N:A, derived for case 2 (assuming $100 \mu \mathrm{m}$ flux for $\mathrm{N}: \mathrm{A} \sim 55 \mathrm{Jy}, \mathrm{N}: \mathrm{B} \sim 11 \mathrm{Jy}$ ), was $1.5 \%$, which lies well above the lower limit value of $0.5 \%$ established for Class 0 objects by André et al. (2000). We point out two additional factors that bolster the argument for the Class 0 status of $\mathrm{N}: \mathrm{A}$ : (1) Its molecular outflow is extremely well collimated, similar to outflows associated with other Class 0 sources (Wolf-Chase et al. 2000). (2) We find that it is not detected in the near-infrared by 2MASS, although the Class I source L1448 IRS 1 is strongly detected in all 2MASS bands $\left(J H K_{S}\right)$. Although fluxes are presented in all bands for a "source" detected by 2MASS within a few arcseconds of L1448N:A, the $J$-band flux is presented as an upper limit, and the $H$-band and $K$-band fluxes suffer photometric confusion. The $2 \mathrm{MASS}$ Quicklook images reveal a bow-shaped knot north of L1448N:A in all three bands and an additional jetlike feature south of L1448N:A in the $K$-band image. Inspection of these features reveals that they correspond to $\mathrm{H}_{2}$ outflow features imaged by Eislöffel (2000). The jetlike feature in the $K$-band image of L1448N:A is part of the blueshifted outflow lobe associated with L1448C, which is located $\sim 1$.'5 to the southeast of the L1448N sources. This outflow was also identified in HIRES-processed IRAS images and high-velocity CO emission (Barsony et al. 1998; Wolf-Chase et al. 2000). The bow-shaped knot that appears just north of L1448N:A in the $J$, $H$, and $K_{s}$ 2MASS images is very prominent in the $\mathrm{H}_{2}$ image. There is no trace of a near-infrared point-source counterpart to L1448N:A in the 2MASS data.
Additional near-infrared data for this source became available during a late revision of this paper: a FLAMINGOS survey of L1448 down to $m_{K_{s}} \sim 17 \mathrm{mag}$ (Tsujimoto et al. 2005). Again, no near-IR counterpart to L1448N:A was found during this survey. However, these same investigators also used Chandra to observe L1448; they detected a weak X-ray source in the general vicinity of N:A. No X-ray emission was detected from any of the other Class 0 sources under discussion in this paper. Tsujimoto et al. (2005) discuss the fact that, given the nondetection in the nearinfrared bands, L1448N:A must have an extremely steep near- to mid-infrared spectral index $(\alpha>3.2)$ and that nearly all known Class I protostars have $\alpha \leq 2$ (the single exception being the peculiar YSO WL 22, with $\alpha=3$ ).

We therefore contend that all currently available data for L1448N:A indicate that it is a bona fide (albeit borderline) Class 0 source; the weak X-ray detection does not rule this out, and all other data support this conclusion.

\subsubsection{Effects of the L1448C Outflow on the L1448N/NW Core}

Using ammonia data, Curiel et al. (1999) report that the highvelocity L1448C outflow has had a significant effect on the northern core, creating a deep "dent" in its southern edge, directly south of the protobinary. Barsony et al. (1998) suggest that the impact of the outflow from L1448C on the dense core fragmented it and actually induced the formation of the L1448N/ NW system.

At first glance, this scenario of outflow-induced fragmentation appears to be at odds with our findings above that N:A is the most evolved Class 0 source in this cloud, while $\mathrm{N}: \mathrm{B}$ is the youngest and most deeply embedded. Of course, if these two sources do not form a gravitationally bound protobinary system, it is unnecessary for them to be coeval. But for the purposes of this discussion we assume along with Terebey \& Padgett (1997), Barsony et al. (1998), Eislöffel (2000), and Wolf-Chase et al. (2000) that N:A and N:B are the bound components of a protobinary. Is it possible for presumably coeval sources, which appear to be physically similar, to travel different evolutionary paths?

One recently proposed explanation for such a dichotomy in the evolutionary status of protobinary components is found in the disintegration of unstable triple or higher order multiple systems; such events may cause disk truncation for some of the components, subsequent episodic outflow activity linked to giant Herbig-Haro flows, and the accelerated evolution of one or more components due to the rapid dissipation of circumstellar envelopes (Reipurth 2000; Reipurth \& Clarke 2001). It is true that most of the young sources in L1448 have been identified as driving sources of parsec-scale Herbig-Haro flows (Bally et al. 1997; Barsony et al. 1998; O’Linger et al. 1999; Eislöffel 2000; Wolf-Chase et al. 2000). However, at 2000 AU separation, with a rotational period on the order of $60,000 \mathrm{yr}$ (Terebey \& Padgett 1997), the L1448N:A, B system does not seem to fit into this particular theoretical paradigm, since a remnant binary from such a former higher multiplicity system is predicted to have a much smaller separation. The L1448N:A, B components appear to be too young to have had time to interact with each other or with L1448NW in any significant way. Furthermore, according to the Reipurth (2000) theory, binary components that are remnants of unstable multiples having undergone ejection events might be expected to have more pronounced differences between their apparent evolutionary stages; e.g., one component may transition abruptly to Class II status, while the other remains in Class 0 or Class I. All of the L1448 sources under discussion 
in this paper still fall within the same evolutionary category, although N:A appears to be close to transitioning to Class I.

We propose an alternative explanation for the (admittedly minor) difference between the protobinary components; this could be nothing more than a chance side effect of the powerful L1448C outflow. Lacking a good understanding of the three-dimensional relative geometry of the sources in this cloud, we are unable to verify our hypothesis, but one possible scenario is that the outflow from L1448C has partially stripped the envelope surrounding $\mathrm{N}$ :A, while leaving N:B deeply embedded. This would allow $\mathrm{N}: \mathrm{A}$ and $\mathrm{N}: \mathrm{B}$ to be coeval sources that formed due to the impact of the L1448C outflow, while explaining the different apparent ages of these objects.

\section{SUMMARY}

We have conducted a mid-infrared imaging survey of known Class 0 protostars in L1448 with MIRLIN at the IRTF, using the $12.5,17.9,20.8$, and $24.5 \mu \mathrm{m}$ filters. Of the five confirmed Class 0 sources in this cluster, we imaged and obtained photometry for three, including a single source that was marginally detected at $24.5 \mu \mathrm{m}$ at the location of the protobinary in L1448 IRS 2. We report upper limits for two undetected objects.

SEDs are presented for all five sources and are used to derive various physical properties of these sources, allowing us to place them on an evolutionary status diagram. The components of the protobinary system L1448N are plotted separately; we find that the source known as L1448N:A lies significantly closer to the Class I portion of the diagram than does its more deeply embedded companion, $\mathrm{N}$ :B. One possible explanation for this dif- ference in apparent "age" may be found in the "stripping" of the envelope surrounding N:A by the L1448C outflow, which is known to be colliding with the northern core.

We thank the IRTF support staff for assistance with the data acquisition. J. O. acknowledges financial support by NASA grants to the Wide-Field Infrared Explorer Project and the Space Infrared Telescope Facility Project at the Jet Propulsion Laboratory, California Institute of Technology. Many thanks to Michael Werner, Vincent Mannings, and our anonymous referee for helpful suggestions that improved this paper immensely. This research has made extensive use of the Abstract Service maintained by the NASA Astrophysics Data System, as well as the Simbad and Vizier databases archived at CDS, Strasbourg, France. This publication makes use of data products from 2MASS, which is a joint project of the University of Massachusetts and the Infrared Processing and Analysis Center, California Institute of Technology, funded by the National Aeronautics and Space Administration and the National Science Foundation. This research has made use of the NASA/ IPAC Infrared Science Archive, which is operated by the Jet Propulsion Laboratory, California Institute of Technology, under contract with the National Aeronautics and Space Administration. Portions of this work were carried out at the Jet Propulsion Laboratory, California Institute of Technology, under contract with the National Aeronautics and Space Administration. Development of MIRLIN was supported by the Jet Propulsion Laboratory's Director's Discretionary Fund, and its continued operation is funded by an SR+T award from NASA's Office of Space Science.
André, P., \& Montmerle, T. 1994, ApJ, 420, 837

André, P., Ward-Thompson, D., \& Barsony, M. 1993, ApJ, 406, 122 2000, in Protostars \& Planets IV, ed. V. Mannings, A. P. Boss, \& S. S. Russell (Tucson: Univ. Arizona Press), 59

Anglada, G., Rodríguez, L., Torrelles, J., Estalella, R., Ho, P., Cantó, J., López, R., \& Verdes-Montenegro, L. 1989, ApJ, 341, 208

Aumann, H. H., Fowler, J. W., \& Melnyk, M. 1990, AJ, 99, 1674

Bachiller, R., André, P., \& Cabrit, S. 1991, A\&A, 241, L43

Bachiller, R., \& Cernicharo, J. 1986a, A\&A, 166, 283 . 1986b, A\&A, 168, 262

Bachiller, R., Cernicharo, J., Martin-Pintado, J., Tafalla, M., \& Lazareff, B. 1990, A\&A, 231, 174

Bachiller, R., Guilloteau, S., Dutrey, A., Planesas, P., \& Martín-Pintado, J. 1995, A\&A, 299, 857

Bally, J., Devine, D., Alten, V., \& Sutherland, R. 1997, ApJ, 478, 603

Barsony, M. 1994, in ASP Conf. Ser. 65, Clouds, Cores, and Low-Mass Stars, ed. D. P. Clemens \& R. Barvainis (San Francisco: ASP), 197

Barsony, M., Ward-Thompson, D., André, P., \& O'Linger, J. 1998, ApJ, 509, 733

Bontemps, S., André, P., Terebey, S., \& Cabrit, S. 1996, A\&A, 311, 858

Chandler, C., \& Richer, J. 2000, ApJ, 530, 851

Ciardi, D., Telesco, C., Williams, J., Fisher, R., Packham, C., Piña, R., \& Radomski, J. 2003, ApJ, 585, 392

Cohen, M., \& Kuhi, L. 1979, ApJS, 41, 743

Curiel, S., Torrelles, J., Rodríguez, L. F., Gómez, J., \& Anglada, G. 1999, ApJ, 527,310

Eislöffel, J. 2000, A\&A, 354, 236

\section{REFERENCES}

Froebrich, D. 2005, ApJS, 156, 169

Greene, T., Wilking, B., André, P., \& Young, E. 1994, ApJ, 434, 614

Guilloteau, S., Bachiller, R., Fuente, A., \& Lucas, R. 1992, A\&A, 265, L49

Hildebrand, R. 1983, QJRAS, 24, 267

Hurt, R., \& Barsony, M. 1996, ApJ, 460, L45

Lada, C. J. 1987, in IAU Symp. 115, Star-Forming Regions, ed. M. Peimbert \& J. Jugaku (Dordrecht: Reidel), 1

_ 1991, in The Physics of Star Formation and Early Stellar Evolution, ed. C. J. Lada \& N. D. Kylafis (Dordrecht: Kluwer), 329

Lada, C. J., \& Wilking, B. A. 1984, ApJ, 287, 610

Looney, L., Mundy, L., \& Welch, W. 2000, ApJ, 529, 477

Neckel, T., \& Staude, H. 1984, A\&A, 131, 200

O'Linger, J., Wolf-Chase, G., Barsony, M., \& Ward-Thompson, D. 1999, ApJ, 515,696

Reipurth, B. 2000, AJ, 120, 3177

Reipurth, B., \& Clarke, C. 2001, AJ, 122, 432

Ressler, M. E., Werner, M. W., van Cleve, J., \& Chou, H. A. 1994, Exp. Astron., 3, 277

Saraceno, P., André, P., Ceccarelli, C., Griffin, M., \& Molinari, S. 1996, A\&A, 309,827

Stahler, S., Shu, F., \& Taam, R. 1980, ApJ, 242, 226

Terebey, S., \& Padgett, D. 1997, in IAU Symp. 182, Herbig-Haro Flows and the Birth of Low-Mass Stars, ed. B. Reipurth \& C. Bertout (Dordrecht: Kluwer), 507

Tsujimoto, M., Kobayashi, N., \& Tsuboi, Y. 2005, AJ, 130, 2212

Volgnau, N., et al. 2002, BAAS, 34, 1216

Wolf-Chase, G. A., Barsony, M., \& O'Linger, J. 2000, AJ, 120, 1467 